\title{
Amnion-Derived Multipotent Progenitor Cells Suppress Experimental Optic Neuritis and Myelitis
}

\author{
Reas S. Khan ${ }^{1} \cdot$ Ahmara G. Ross $^{1} \cdot$ Keirnan Willett $^{1} \cdot$ Kimberly Dine $^{1} \cdot$ Rick Banas $^{2} \cdot$ Larry R. Brown $^{2}$. \\ Kenneth S. Shindler ${ }^{1,3}$ (B)
}

Accepted: 9 October 2020 / Published online: 16 October 2020

(C) The American Society for Experimental NeuroTherapeutics, Inc. 2020

\begin{abstract}
The human amnion has been used for decades in wound healing, particularly burns. Amnion epithelial cells (AECs) have been the focus of extensive research based on their possible pluripotent differentiation ability. A novel, cultured cell population derived from AECs, termed human amnion-derived multipotent progenitor (AMP) cells, secrete numerous cytokines and growth factors that enhance tissue regeneration and reduce inflammation. This AMP cell secretome, termed ST266, is a unique biological solution that accumulates in eyes and optic nerves following intranasal delivery, resulting in selective suppression of optic neuritis in the experimental autoimmune encephalomyelitis (EAE) model of multiple sclerosis, but not myelitis at the administered dose. We tested the hypothesis that systemic AMP cell administration could suppress both optic neuritis and myelitis in EAE. Intravenous and intraperitoneal administration of AMP cells significantly reduced ascending paralysis and attenuated visual dysfunction in EAE mice. AMP cell treatment increased retinal ganglion cell (RGC) survival and decreased optic nerve inflammation, with variable improvement in optic nerve demyelination and spinal cord inflammation and demyelination. Results show systemic AMP cell administration inhibits RGC loss and visual dysfunction similar to previously demonstrated effects of intranasally delivered ST266. Importantly, AMP cells also promote neuroprotective effects in EAE spinal cords, marked by reduced paralysis. Protective effects of systemically administered AMP cells suggest they may serve as a potential novel treatment for multiple sclerosis.
\end{abstract}

Keywords Neuroprotection $\cdot$ Multiple sclerosis $\cdot$ Experimental autoimmune encephalomyelitis $\cdot$ Optic neuritis $\cdot$ Retinal ganglion cell $\cdot$ Amnion-derived multipotent progenitor cell. · ST266

Rick Banas is deceased. This paper is dedicated to his memory.

Electronic supplementary material The online version of this article (https://doi.org/10.1007/s13311-020-00949-9) contains supplementary material, which is available to authorized users.

Kenneth S. Shindler

Kenneth.Shindler@uphs.upenn.edu

1 Department of Ophthalmology, University of Pennsylvania, Philadelphia, PA, USA

2 Noveome Biotherapeutics, Inc., Pittsburgh, PA, USA

3 F.M. Kirby Center for Molecular Ophthalmology, Department of Ophthalmology, University of Pennsylvania Scheie Eye Institute, Stellar-Chance Laboratories, 3rd Floor, 422 Curie Blvd, Philadelphia, PA 19104, USA

\section{Introduction}

Multiple sclerosis (MS) is a progressive autoimmune nervous system disease that is associated with chronic inflammation, demyelination, and neurodegeneration of the central nervous system (CNS) [1]. According to recent studies, MS affects nearly 2.5 million people worldwide with an average age of incidence of about 32 years, and the disease is twice as common in women as in men [2]. Plaques developed as a result of robust inflammatory infiltration combined with demyelination in the brain, spinal cord, and optic nerves are the unique pathological features of MS [3]. Optic neuritis (ON), an inflammatory demyelinating disease of the optic nerve, is one of the most common symptoms of the relapsing-remitting form of MS and for some patients, ON is the initial symptom of MS [4]. Loss of retinal ganglion cells (RGCs), the neurons of the 
optic nerve, is increasingly recognized as a correlate of the visual disability in MS and experimental models of MS and ON $[5,6]$.

Current medications used to treat MS slow or modify the course of the disease, make relapsing inflammatory attacks less frequent, slow the progression of the disease, and manage MS symptoms to enhance the quality of life of patients. High doses of corticosteroids that suppress multiple inflammatory pathways in the body are used to manage acute exacerbations or relapses in MS patients [7]. However, there is limited evidence that steroid therapy reduces tissue damage or induces a difference in the degree of clinical recovery after a relapse [8] and there is controversy over whether steroids reduce or delay subsequent relapses [9-11]. Corticosteroid treatment has limited effects in preventing axonal and RGC loss in MS [12, 13] and studies with animal models of MS show that high doses of corticosteroids may even result in exacerbation of neuronal apoptosis [14]. Therefore, new therapies targeting the neurodegenerative aspects of MS are needed.

Recent investigations have revealed stem cell-based therapies may be effective in MS treatment $[15,16]$. Isolated stem cell populations can be expanded in culture to generate large numbers of cells, and the effectiveness and safety profiles make some stem cell populations potential candidates for MS treatment [17]. Amnion epithelial cells (AECs) have shown tremendous immunosuppressive potential and protect mice from developing signs of experimental autoimmune encephalomyelitis (EAE), a commonly used animal model of MS [18]. Proprietary amnion-derived multipotent progenitor (AMP) cells are a unique subpopulation of nonimmunogenic AECs which are selected based on their ability to attach and proliferate in serum-free culture conditions [19]. AMP cells are well characterized and have been shown to be immunomodulatory, facilitated by cell-to-cell contact with target immune cells and by secretion of anti-inflammatory molecules [19, 20]. ST266, a novel secretome derived from proprietary GMP cultured human AMP cells, contains hundreds of proteins and other biological factors and has shown significant RGC neuroprotective effects in EAE following intranasal delivery [21]. Intranasally administered ST266 selectively accumulates in the eye and optic nerve and suppresses optic neuritis lesions in the EAE model of MS. However, at the administered dose and dosing schedule, there was no effect on spinal cord lesions [21]. Direct neuroprotective effects of systemically administered AMP cells on optic nerve and spinal cord degeneration have not been studied. In the present study, we investigated the neuroprotective ability of systemic administration of AMP cells on clinical disease progression and histopathology of optic neuritis and myelitis in EAE mice.

\section{Methods}

\section{Animals}

Six-week-old female C57BL/6J mice obtained from The Jackson Laboratory (Bar Harbor, ME, USA), were housed in specific pathogen-free animal facilities and transferred to biosafety level 2 conditions for injection of human cells at the University of Pennsylvania. Mice were housed in a $12 \mathrm{~h}$ light/ dark cycle at an ambient temperature of $22{ }^{\circ} \mathrm{C}$ and fed standard rodent chow and water ad libitum. All animal experiments were approved by and conformed to Institutional Animal Care and Use Committee at the University of Pennsylvania guidelines. All applicable international, national, and institutional guidelines for the care and use of animals were followed.

\section{Induction and Scoring of EAE}

EAE was induced as previously described [21, 22]. Briefly, 8-week-old female C57BL/6J mice were anesthetized with isoflurane and injected subcutaneously on the upper and lower back with a total of $200 \mu \mathrm{g}$ of myelin oligodendrocyte glycoprotein (MOG) peptide $\left(\mathrm{MOG}_{35-55}\right.$; Genscript, Piscataway, NJ, USA) emulsified in Complete Freund's Adjuvant (Difco, Detroit, MI, USA) containing $2.5 \mathrm{mg} / \mathrm{ml}$ Mycobacterium tuberculosis (Difco). Control mice were injected with an equal volume of phosphate buffer saline (PBS) instead of MOG and Complete Freund's Adjuvant. All animals received $200 \mathrm{ng}$ pertussis toxin (List Biological, Campbell, CA, USA) in $0.1 \mathrm{ml}$ PBS by intraperitoneal injection at $0 \mathrm{~h}$ and $48 \mathrm{~h}$ after immunization. Severity of EAE was scored using a previously published [21, 23] 5-point scale: $0=$ no disease; $0.5=$ partial tail paralysis; $1.0=$ tail paralysis or waddling gait; $1.5=$ partial tail paralysis and waddling gait; $2.0=$ tail paralysis and waddling gait; $2.5=$ partial limb paralysis; $3.0=$ paralysis of one limb; $3.5=$ paralysis of one limb and partial paralysis of another; $4.0=$ paralysis of two limbs; $4.5=$ moribund state; $5.0=$ death.

\section{AMP Cell Administration}

AMP cells (Noveome Biotherapeutics, Inc., Pittsburgh, PA, USA) were transported frozen and thawed in proprietary STM100 media (Hyclone, Logan, UT, USA). The cells were washed once with STM100 media, centrifuged and pellets were resuspended in DNAse-Heparin- $\mathrm{MgCl}_{2}$ vehicle solution (Worthington Biochemical Cooperation, Lakewood, NJ, USA; Sigma-Aldrich, St. Louis, MO, USA) for a second wash. The cells were resuspended in DNAse-Heparin$\mathrm{MgCl}_{2}$ vehicle solution, counted using a hemocytometer and diluted to the desired cell number in a $200 \mu \mathrm{L}$ injection volume. The DNAse-Heparin- $\mathrm{MgCl}_{2}$ vehicle solution is used to 
alleviate the chances of acute pulmonary embolism in mice. AMP cell treatment groups of mice were injected by intravenous (IV) or intraperitoneal (IP) administration. Untreated EAE mice received an equal volume of vehicle solution only. $1 \times$ dosage groups received $2 \times 10^{6}$ AMP cells by IP or IV injection on day 9 after immunization (p.i.), and $3 \times$ dosage groups received $1 \times 10^{6}$ AMP cells by IP or IV injections repeatedly on three separate days: day 9, 12 , and 15 p.i.

\section{Measurement of Optokinetic Responses}

Optokinetic responses (OKR) were measured using OKR apparatus and visuomotor software (OptoMotry; Cerebral Mechanics, Inc., Lethbride, AB, Canada) to estimate visual function, as in prior studies [24, 25]. Briefly, each mouse was placed on a circular platform in the apparatus and monitored for the ability to visualize rotating sinusoidal gratings with different special frequencies projected on a virtual sphere displayed onto four screens to evoke OKR visual stimuli. OKR function is determined by the highest spatial frequency at which mice track a $100 \%$ contrast grating. Data are reported as cyc/deg.

\section{Quantification of Retinal Ganglion Cell Survival and Axonal Numbers in the Retina}

RGCs were immunolabeled with Brn3a antibody in flatmounted retinas and counted as in prior studies [21, 22]. Briefly, mice were sacrificed at day 42 p.i.; eyes were isolated, fixed for $1 \mathrm{~h}$ at room temperature in $4 \%$ paraformaldehyde, and retinas were dissected and prepared as flattened whole mounts. Specimens were then washed 3 times with PBS and permeabilized in $0.5 \%$ Triton X-100 in PBS by freezing for $15 \mathrm{~min}$ at $-70{ }^{\circ} \mathrm{C}$. Specimens were then incubated in a humidified chamber overnight at $4{ }^{\circ} \mathrm{C}$ with 1:2000 rabbit antiBrn3a antibody (RGC marker, SYSY Synaptic Systems, Goettingen, Germany) and 1:200 anti-mouse RT-97 antibody (DSHB, Univ. Iowa) diluted in a blocking buffer containing $2 \%$ bovine serum albumin and $2 \%$ Triton X-100. The specimens were washed three times in PBS and then incubated for $1 \mathrm{~h}$ at room temperature with anti-rabbit Alexa Fluor 488 and anti-mouse Alexa Fluor 594-conjugated secondary antibodies (Thermo Fisher Scientific, Waltham, MA, USA), each diluted 1:500 in blocking buffer. After 5 washing steps with PBS, the retinas were mounted vitreous side up on slides in Fluoromount-G mounting medium (Southern Biotech, Birmingham, AL, USA). Photographs of the Brn3a-positive RGCs were taken at $\times 40$ magnification in 12 standard fields: $1 / 6,3 / 6$, and $5 / 6$ of the retinal radius from the center of the retina in each quadrant using a fluorescent microscope (Nikon, Melville, NY, USA) and counted by a masked investigator using image analysis software (Image-Pro Plus 5.0;
Media Cybernetics, Silver Spring, MD). Data shown represent the average of the total number of RGCs counted in all 12 fields of each retina (covering a total area of $4.8 \times 10^{5} \mathrm{um}^{2}$ ). For axonal quantification in the retina, photographs of RT-97 stained axonal fibers were taken at $\times 40$ magnification in 4 standard fields (top, bottom, left, and right) at $1 / 6$ of the retinal radius. The axonal fiber numbers were counted by a masked investigator. Data shown represent the average of the total number of axons counted in all 4 fields of each retina.

\section{Evaluation of Spinal Cord Inflammation and Demyelination}

Inflammation and demyelination in the spinal cord were assessed by previously published methods [26, 27]. On day 42 p.i., mice were transcardially perfused and spinal cords were isolated and post-fixed in $4 \%$ paraformaldehyde for $48 \mathrm{~h}$. The spinal cords were then extracted, processed, and embedded in paraffin, and 5- $\mu \mathrm{m}$ coronal sections were made. Inflammation was assessed by staining with hematoxylin and eosin (H\&E) and examined by light microscopy. The presence of inflammatory cell infiltrates and the relative degree of inflammation was scored by a masked investigator using a 0-3 point scale: $0=$ no inflammation; $1=$ mild inflammation (less than 5 small foci of white matter inflammatory cell infiltration across all sections); $2=$ moderate inflammation (5-9 small foci of inflammation, or 1-2 large areas of inflammation); $3=$ severe inflammation (more than 10 small foci or more than two large areas of inflammation). Three sections were examined from each of three spinal cord levels (cervical, thoracic, and lumbar) for each mouse. IBA1 (a marker for microglia/ macrophages) staining was used to further evaluate the presence of inflammatory cell infiltration in the spinal cord. Briefly, antigen retrieval was done on deparaffinized and rehydrated spinal cord specimens by heating at $95^{\circ} \mathrm{C}$ in Vector antigen unmasking solution (Vector Labs, Burlingame, CA, USA) for $15 \mathrm{~min}$. Blocking was done with blocking reagent from Vectastain Elite Avidin/Biotin Complex kit (ABC; Vector Labs). Sections were incubated in 1:200 concentration of rabbit anti-IBA1 antibody (WAKO, Richmond, VA, USA) diluted in blocking buffer and incubated at $4{ }^{\circ} \mathrm{C}$ overnight. After three washes with PBS, sections were incubated with goat biotinylated antirabbit secondary antibody (Invitrogen, Carlsbad, CA, USA) for $2 \mathrm{~h}$ at room temperature. Vectastain Elite $\mathrm{ABC}$ kit and DAB (diaminobenzidine) substrate kit (Vector Labs) was used to perform Avidin/Biotin Complex detection according to the manufacturer's instructions. Sections were examined by light microscopy and photographs were taken at $\times 10$ magnification. To assess demyelination, spinal cord coronal sections were deparaffinized, rehydrated, and were stained with Luxol Fast blue (LFB) using a previously established protocol 
$[26,28]$. Areas of demyelination were examined by light microscopy and quantified by a masked investigator using a $0-3$ point scale: $0=$ no demyelination; $1=$ rare foci of demyelination; 2 = a few foci of demyelination; and $3=$ large (confluent) areas of demyelination. To confirm demyelination, additional spinal cord sections were stained with antimyelin basic protein (MBP) antibodies and scored by using the same scale. Briefly, $5 \mu \mathrm{M}$ sections were subjected to antigen retrieval using boiling citrate-based antigen unmasking solution (Vector Laboratories, Burlingame, CA, USA) after deparaffinization and rehydration. Non-specific binding was blocked by incubation with blocking reagent from Vectastain Elite ABC kit (Vector Laboratories) with $0.2 \%$ Triton X-100. The specimens were then incubated overnight at $4{ }^{\circ} \mathrm{C}$ using a primary mouse anti-MBP antibody (Biolegend, San Diego, CA, USA) diluted 1:500 in blocking buffer. The sections were then washed four times in PBS, incubated with biotinylated anti-mouse secondary antibody (Vector Laboratories) for $30 \mathrm{~min}$ at $37^{\circ} \mathrm{C}$, then washed with PBS four times followed by incubation with $\mathrm{ABC}$ reagent (Vector Laboratories) for another $30 \mathrm{~min}$ at $37^{\circ} \mathrm{C}$. The specimens were developed with $\mathrm{DAB}$ (diaminobenzidine) substrate from the DAB substrate kit (Vector Laboratories) followed by washing in running water to stop the DAB reaction. The specimens were then dehydrated, mounted using Refrax mounting medium and scored by a masked investigator using the same scale as used for LFB staining.

\section{Evaluation of Optic Nerve Inflammation and Demyelination}

Mice were sacrificed at day 42 p.i., optic nerves were isolated, fixed in $4 \%$ paraformaldehyde, embedded in paraffin and $5 \mu \mathrm{m}$ longitudinal sections were cut using a microtome (Leica Biosystems, Illinois, USA). To evaluate inflammation, sections were stained with $\mathrm{H} \& \mathrm{E}$ as in prior studies [21, 22], examined by light microscopy and scored using a 4 point scale: $0=$ no infiltration; $1=$ mild cellular infiltration of the optic nerve or optic nerve sheath (focal inflammation involving less than $25 \%$ of the length of the optic nerve as viewed by a masked investigator); $2=$ moderate infiltration $(25-50 \%$ of optic nerve area involved); $3=$ severe infiltration $(50-75 \%)$; and $4=$ massive infiltration $(>75 \%)$. IBA1 staining was also used to evaluate the presence of inflammatory cell infiltration in the optic nerve using the same scoring scale, with staining performed as described for the spinal cord. To detect demyelination, optic nerve sections were stained with LFB and quantified on a 0-3-point relative scale by a masked investigator as previously $[21,22]$ described: $0=$ no demyelination; $1=$ scattered foci of demyelination; 2 = prominent foci of demyelination; and $3=$ large (confluent) areas of demyelination. The entire length of each optic nerve section was examined.

\section{Statistics}

Evaluation of ascending paralysis by EAE scores and of visual function by OKR thresholds over time was compared using ANOVA of repeated measures followed by Tukey's post hoc comparisons between each group. RGC counts, final OKR scores, spinal cord and optic nerve inflammation scores, and spinal cord and optic nerve demyelination scores were compared by one-way ANOVA with Tukey's post hoc comparisons between treatment groups. Comparisons of eye and visual outcomes (OKR scores, RGC counts, and optic nerve inflammation and demyelination) were performed using measurements from the right eye only. All statistical comparisons were calculated using Graph Pad Prism (GraphPad Software, San Diego, CA). $p$ values less than 0.05 were considered significant.

\section{Results}

\section{AMP Cells Reduce Ascending Paralysis Induced by Spinal Cord EAE Disease}

Severe inflammation, demyelination and axonal loss in the spinal cord leading to progressive hind limb paralysis is the hallmark of EAE, an animal model of MS that recapitulates human MS pathophysiology [29, 30]. Mice were immunized with MOG peptide on day 0 to induce EAE, whereas control mice were sham-immunized with PBS. Mice receiving treatment received $2 \times 10^{6}$ AMP cells by a single IV or IP injection (AMP $\times 1$ ) on day 9 p.i., or alternatively received $1 \times 10^{6}$ AMP cells in three IV or IP injections (AMP $\times 3$ ) given once each on days 9, 12, and 15 p.i. as diagrammed (Fig. 1a). MOG injection in $\mathrm{C} 57 \mathrm{BL} / 6 \mathrm{~J}$ mice resulted in chronic progressive paralytic disease with clinical signs of EAE disease developing around day 15 p.i. and progressed until mice were euthanized on day 42 p.i. (Fig. 1b). EAE mice showed a significant increase $(p<0.001)$ in the clinical score compared to control mice. EAE mice treated with $2 \times 10^{6}$ AMP cells IV on day 9 p.i. showed a significant attenuation of EAE scores $(p<0.001)$ as compared with vehicle (PBS) treated EAE mice. $3 \times$ dosage of $1 \times 10^{6}$ AMP cells administrated by IV on days 9,12 and 15 p.i. also showed a significant $(p<0.001)$ reduction in clinical scores. Mice that received a single dose of $2 \times 10^{6}$ AMP cells IP on day 9 p.i. also showed a significant $(p<0.001)$ attenuation of clinical scores, whereas $3 \times$ IP administration of $1 \times 10^{6}$ AMP cells on days 9,12 , and 15 p.i. only showed a non-significant trend toward reduced clinical scores in EAE mice (Fig. 1b).

\section{AMP Cells Modify Spinal Cord Demyelination}

Lesions in the spinal cord characterized by inflammatory cell infiltration and demyelination are features of EAE shown previously by us and others [26, 27, 31-33]. To evaluate 
Fig. 1 AMP cells suppress EAE induced ascending paralysis. a Experimental design is diagrammed: A single dose $(1 \times)$ of $2 \times 10^{6}$ AMP cells were given by intravenous (IV) or intraperitoneal (IP) injection on day 9 after immunization (p.i.). Additional groups of EAE mice were treated with 3 doses $(3 \times)$ of $1 \times 10^{6}$ AMP cells given IP or IV on days 9, 12, and 15 p.i. OKR was measured at baseline (day 0 ) before immunization, and weekly thereafter. EAE mice were scored daily for signs of ascending paralysis, and mice were euthanized on day 42 p.i. b C57BL/6 mice were immunized with MOG on day 0 , and daily EAE clinical scoring was done based on a 5 point scale. The clinical symptoms of EAE began by day $14 \mathrm{p}$.i. and progressed through day 42 in vehicle (PBS)-treated EAE mice $(N=6)$. $1 \times$ AMP cells given by IV administration $(N=7)$, and $3 \times \mathrm{AMP}$ cell treatments given by IP $(\mathrm{N}=$ 6) or IV $(N=9)$ injection all showed a significant $(* * * p<0.001)$ attenuation of EAE clinical scores as compared with vehicle-treated EAE mice, whereas $1 \times$ treatment with AMP cells by IP injection $(N=6)$ did not significantly reduce EAE scores. Data represent mean \pm SEM a
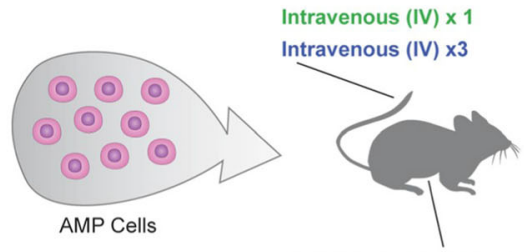

Intra peritoneal (IP) $\times 1$ Intra peritoneal (IP) $\times 3$

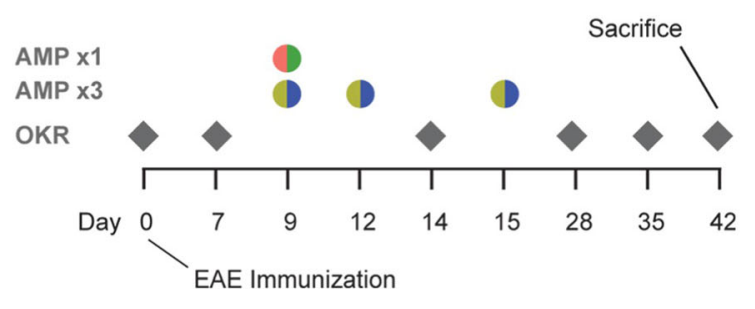

b

AMP Cells Suppress EAE Paralysis

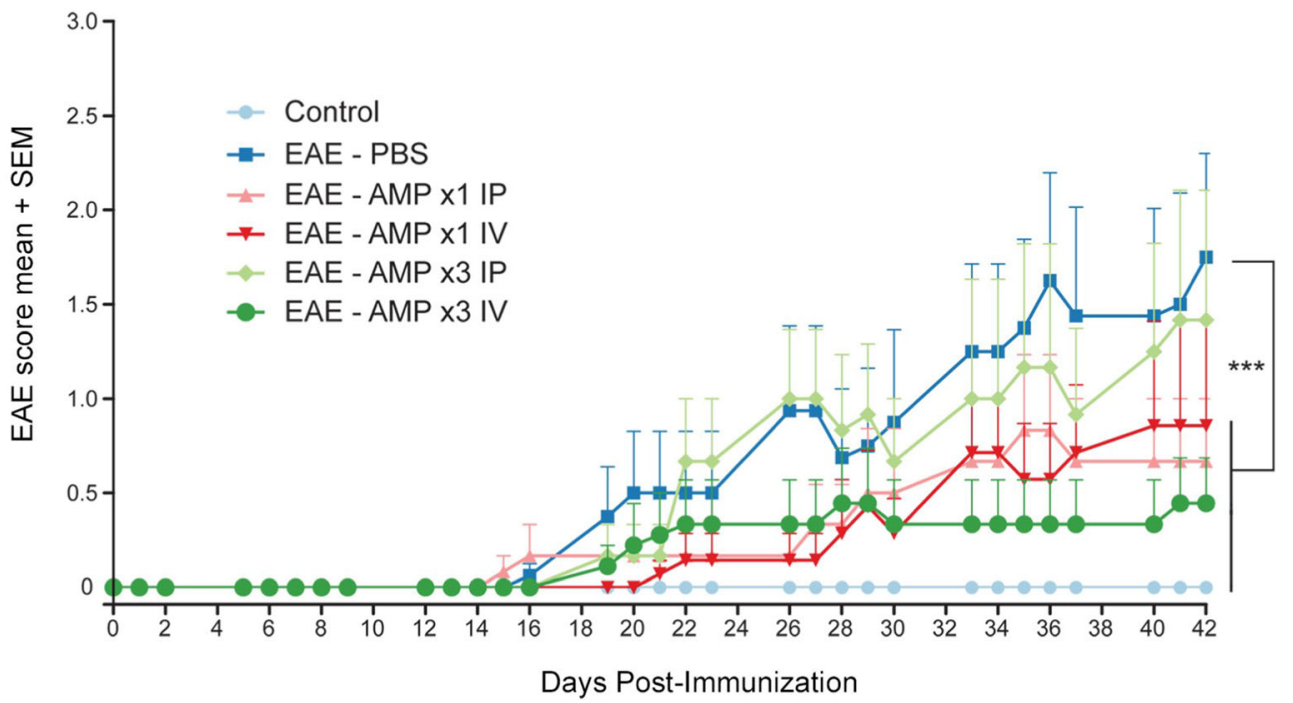

inflammation in the spinal cord, all groups of mice were euthanized on day 42 p.i., and $5 \mu \mathrm{M}$ coronal sections of spinal cords were stained with H\&E and IBA1 (marker of microglia/ macrophages). Consistent with prior findings, inflammation evident by increased H\&E and IBA1 staining in the spinal cord was observed in EAE mice as compared with control mice (Fig. 2a, b). Treatment with $1 \times$ AMP cells by IV or IP injection, and $3 \times$ AMP cells by IV injection, showed a trend toward decreased inflammation scores but it was not significant. To evaluate demyelination, spinal cord sections were stained with LFB and MBP. In agreement with our prior studies, increased demyelinating lesions were observed in the spinal cord of EAE mice as compared with control mice, and $1 \times$ $(p<0.05)$ or $3 \times(p<0.01)$ IV AMP cells significantly decreased the level of demyelination measured by LFB staining as compared with vehicle (PBS) treated EAE mice (Fig. 2c, d), $1 \times$ administration of AMP cells by IP injection showed a non- significant trend toward decreased demyelination scores. Demyelination scored on MBP stained spinal cord sections confirmed similar trends and differences in EAE mice treated with AMP cells, with the addition of the $1 \times$ IP AMP cell treatment group showing a statistical improvement as compared with vehicle-treated mice (average demyelination scores of $1.88 \pm 0.40(N=8$ vehicle-treated mice) versus $0.67 \pm$ $0.33^{*}(N=61 \times$ IP AMP cell-treated mice $) ; 0.71 \pm 0.29^{*}$ $(N=71 \times$ IV $) ; 1.00 \pm 0.52(N=63 \times$ IP $)$; and $0.33 \pm 0.17^{* *}$ $(N=93 \times \mathrm{IV}))$. Scores represent mean $\pm \mathrm{SEM}, * p<0.05$ and $* * p<0.01$ versus vehicle-treated mice.

\section{AMP Cells Suppress Optic Nerve Inflammation and Demyelination}

Inflammation, demyelination, and neurodegeneration in the optic nerve are key pathologic features implicated in EAE 
and MS optic neuritis [34-38]. To evaluate inflammation in the optic nerve, control, EAE, and AMP cell-treated mice were sacrificed on day 42 p.i. $5 \mu \mathrm{M}$ longitudinal optic nerve sections were stained with H\&E and IBA1 (Fig. 3a, b). a

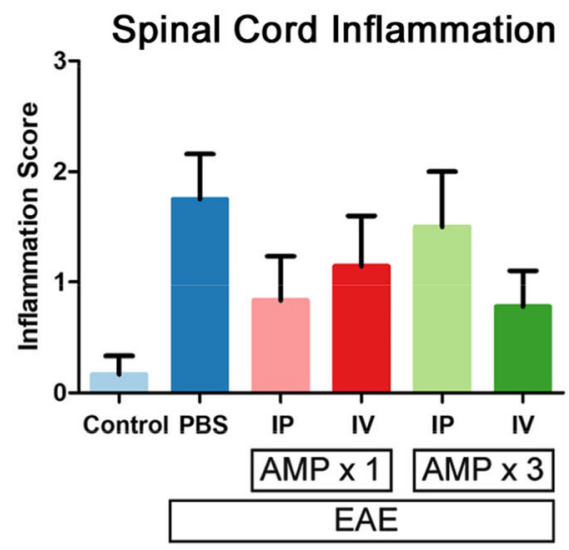

c Spinal Cord Demyelination

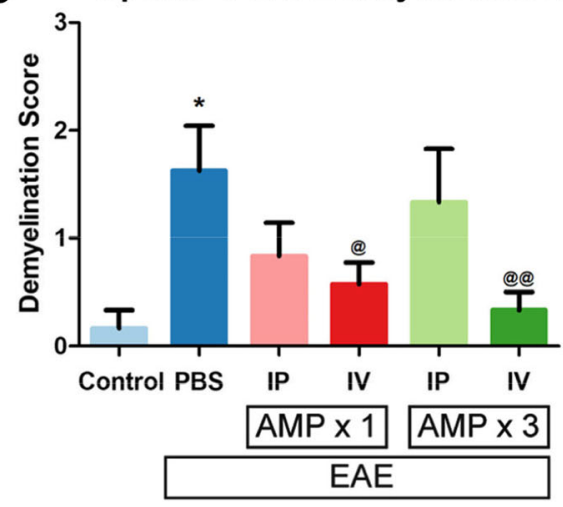

b IBA1+ Microglia/Macrophages in Spinal Cord

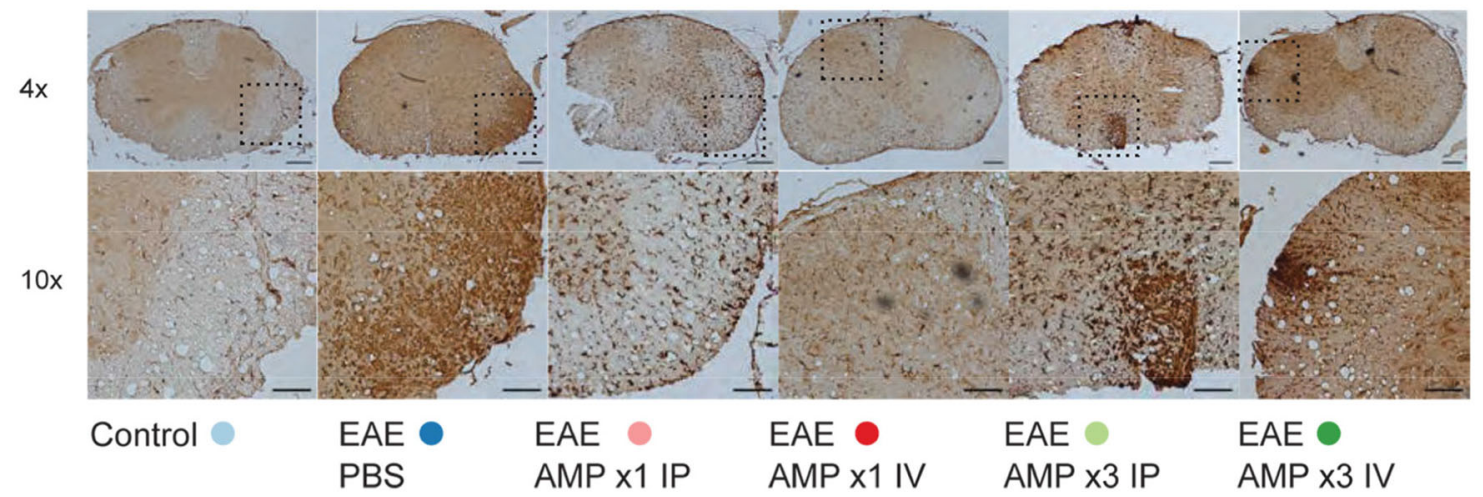

d LFB Staining of Spinal Cord Myelin

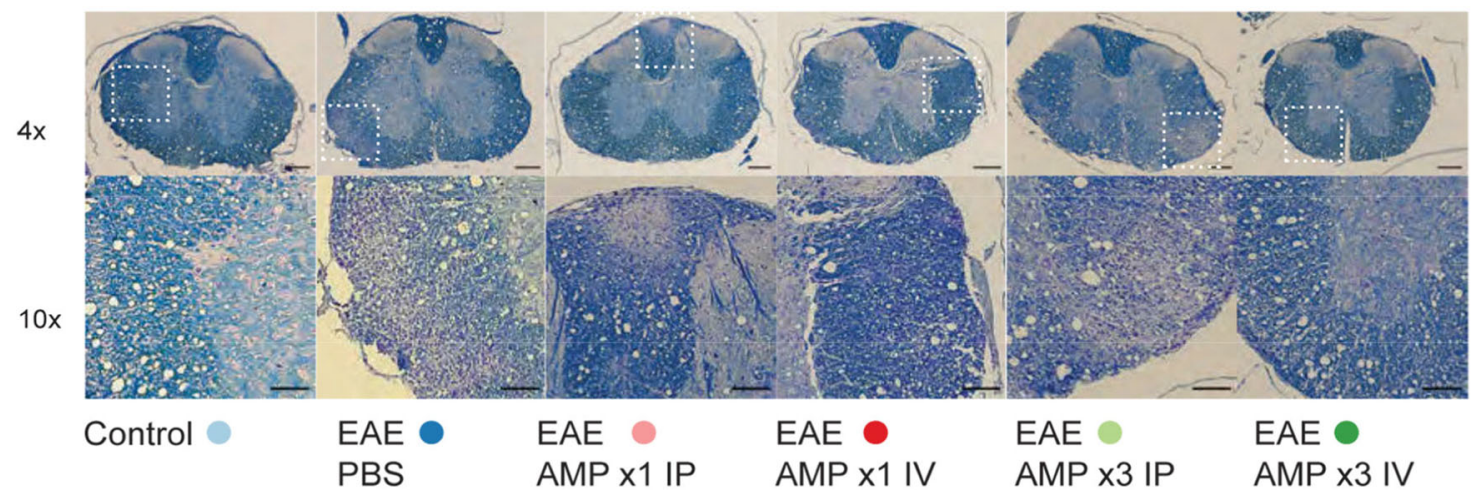

Fig. 2 AMP cells reduce spinal cord demyelination. a Antineuroinflammatory effects of systemic AMP cell administration was evaluated by $\mathrm{H} \& \mathrm{E}$ staining and scoring using a 3 -point scale. $1 \times \mathrm{IP}(N=6)$ or IV $(N=7)$ injection, and $3 \times$ IV $(N=9)$ injection, of AMP cells showed strong trends toward decreasing spinal cord inflammation as compared with vehicle (PBS) treated EAE mice $(N=8)$, but differences were not significant. b IBA1 immunostaining of spinal cord cross sections was performed to detect the presence of microglia/macrophages. Similar to levels of inflammation detected on H\&E staining, representative images show few IBA1+ cells in one control mouse spinal cord, and numerous

IBA1+ present in a vehicle-treated EAE mouse spinal cord. Varying levels of IBA1+ cells were observed in EAE mice treated with AMP cells. c Myelin in spinal cord sections was stained with LFB and levels of demyelination were scored on a 3 point scale. Vehicle-treated EAE mice have significantly more myelin loss than control $(N=6)$ mice (*p, 0.05$)$, and treatment with $1 \times\left({ }^{@} p<0.05\right)$ or $3 \times\left({ }^{@} @ p<0.01\right)$ AMP cells by IV injection significantly reduced the level of spinal cord demyelination as compared with vehicle-treated EAE mice. d Images show LFB staining in one representative mouse spinal cord from each treatment group. Data represent mean \pm SEM. Scale bars $4 \times: 100 \mu \mathrm{M}, 10 \times: 50 \mu \mathrm{M}$ 
Significant optic nerve inflammation developed in EAE mice as compared with control mice. All four AMP cell treatments ( $1 \times$ or $3 \times$ treatment by IP or IV injections) significantly decreased inflammation in the optic nerves as compared with vehicle (PBS) treated mice as shown by IBA1 staining (Fig. 3a, b), with similar results found on H\&E staining (average inflammation scores of $1.88 \pm$ $0.30(N=8$ vehicle-treated mice $)$ versus $1.17 \pm 0.31$ $(N=61 \times$ IP AMP cell-treated mice $) ; 0.43 \pm 0.20 * *$ $(N=7 \quad 1 \times \mathrm{IV}) ; 0.67 \pm 0.33 *(N=63 \times \mathrm{IP}) ;$ and $0.67 \pm$ 0.24* $(N=93 \times$ IV $))$. Scores represent mean \pm SEM, $* p<0.5$, and $* * p<0.01$ versus vehicle-treated mice. Optic nerve demyelination, assessed by LFB staining (Fig. 3c, d), showed a significant $(p<0.01)$ increase in the demyelination score in EAE mice as compared with control mice. Treatment with $1 \times$ AMP cells by IV $(p<0.01)$ or IP $(p<0.05)$ injection, and $3 \times$ AMP cells by IV injection $(p<0.05)$, each showed a significant attenuation in demyelination as compared with vehicle (PBS) treatment.

\section{AMP Cells Attenuate Visual Dysfunction, RGC Loss, and Axonal Degeneration}

OKR responses were measured to evaluate visual function as in prior studies of optic neuritis [21, 22]. EAE mice demonstrated significant $(p<0.01)$, progressive decrease in OKR scores over time across the 6-week experiment as compared with control mice by ANOVA of repeated measures, and a severe $(p<0.001)$ decrease in OKR responses by day 42 p.i. (Fig. 4a, b). Decreasing visual function over time was significantly attenuated by $3 \times$ treatment with AMP cells by IP and IV injection $(p<0.01)$ as well as $1 \times$ IV AMP cell treatment $(p<0.05)$, while $1 \times$ treatment with AMP cells by IP injection led to a strong but non-significant trend of improved OKR responses as compared with vehicle (PBS) treated EAE mice (Fig. 4a). By day 42 p.i., OKR scores showed that treatment with AMP cells induced significant preservation of visual function in all treatment groups as compared with vehicle (PBS) treated EAE mice (Fig. 4b). To evaluate RGC loss, retinas isolated at day 42 p.i. were immunostained with Brn3a antibodies. RGC counts showed that EAE induced significant RGC loss as compared with control mice, and treatment with AMP cells in all four treatment groups led to a significant preservation of RGCs as compared with vehicle (PBS) treated EAE mice (Fig. 4c, d). To evaluate axonal loss in the retina, retinas were stained with Neurofilament RT-97 antibodies. Retinal axonal counts showed that EAE induced significant $(p<0.001)$ axonal loss as compared with control mice, and treatment with AMP in all four treatment groups led to a significant preservation of axons as compared with vehicle (PBS) treated EAE mice (Fig. 4e).

\section{Discussion}

Our results demonstrate that peripheral injection of AMP cells at varying IP and IV doses significantly attenuates the visual dysfunction, RGC loss, and optic nerve inflammation and demyelination that occurs during EAE optic neuritis, and similarly suppresses spinal cord demyelination and associated paralysis. Our prior studies with ST266, the secretome of AMP cells, showed that when given intranasally ST266 proteins accumulated at therapeutic levels in optic nerve and vitreous and preserved RGC function by ameliorating RGC loss, demyelination and inflammation during experimental optic neuritis, but had no effect on spinal cord disease at the administered dose. This may be due to suboptimal dosing or the preferential accumulation of ST266 in ocular tissues as compared with other regions. The neuroprotective effects observed in the present study are consistent with the similar optic nerve neuroprotective effects observed previously with intranasal ST266. Interestingly, the present study also showed a significant reduction in ascending paralysis from spinal cord EAE disease, suggesting that systemically administered AMP cells secrete similar factors as those present in ST266, either systemically to modulate peripheral immune system responses, or locally after migration into distal portions of the CNS to exert effects in distinct regions. Previous studies have shown that the immunomodulatory effect of AMP cells is dependent on cell-to-cell contact, while the cells are largely non-immunogenic due to a lack of MHC class II antigens [19, 20]. One of the unique characteristics of AMP cells is that they express the tissue-restricted, nonclassical HLA class I antigen HLA-G [19, 20, 39-41]. HLA-G has been shown to have substantial immunomodulatory functions [42-44]. Furthermore, the immunomodulatory pathway mediated by AMP cells appears to be through their impact on the maturation of monocyte-derived dendritic cells [20]. While daily intranasal ST266 administration may serve as a potential focal therapy for the eye and optic nerve, current results suggest limited repeat doses, or even single peripheral administration of AMP cells, has tremendous therapeutic potential for a broad spectrum of neuroprotective activity by attenuating both optic neuritis and transverse myelitis, and thus warrants further investigation as a novel therapy for MS. Treatment was initiated 9 days after immunization, the earliest time in which optic nerve inflammation has been found in our prior studies, with subsequent treatments in groups receiving three doses given on days 12 and 15 , the latter being the day by which optic nerve inflammation reaches maximal levels [25]. Thus, these represent the earliest potential clinically relevant time points when patients may be developing eye pain or early visual symptoms, and the positive results here suggest future studies with treatment initiated at even later time points will be interesting. 
The current results are consistent with prior studies showing immunosuppressive and therapeutic effects of human amniotic cells in EAE [45, 46]. A major advantage of the novel AMP cells used in the current study is that they are produced and cultured under Current Good Manufacturing Practice (cGMP) conditions, and these cells are used to produce the otherwise acellular ST266 secretome currently in clinical trials for treating other disorders (clinical trials.gov.: NCT03901781,
AMP Cells Reduce Optic Nerve Inflammation

a

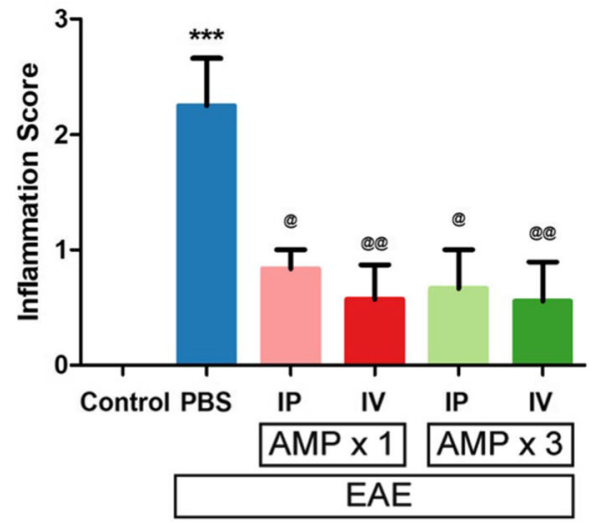

\section{AMP Cells Reduce Optic Nerve Demyelination}

c

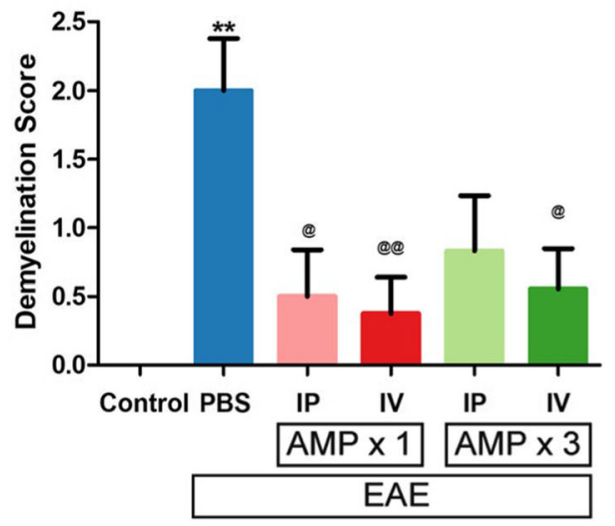

b

AMP Cells Reduce Optic Nerve Inflammation

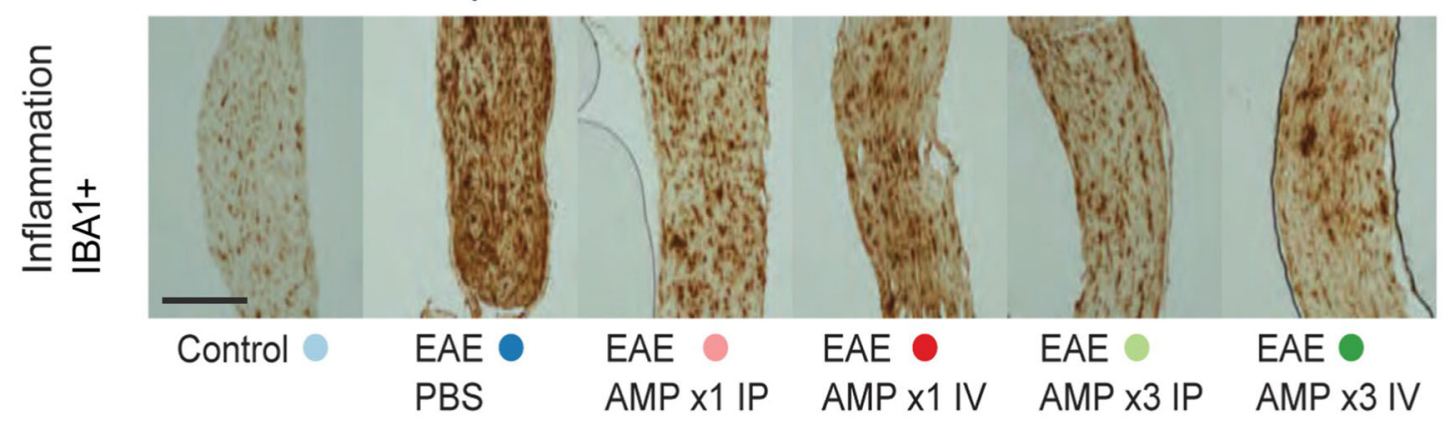

C

AMP Cells Reduce Optic Nerve Demyelination
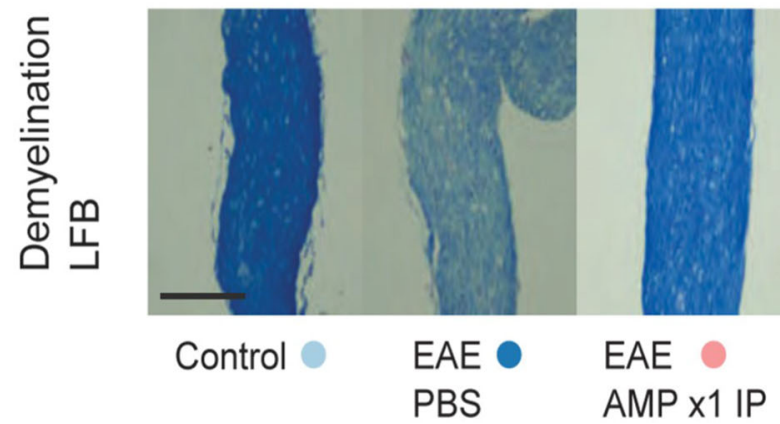

Fig. 3 AMP cells suppress optic nerve inflammation and demyelination. a Immunomodulatory effects of systemic AMP cells in the optic nerve was evaluated by IBA1 staining and scored using a 4 point scale. Vehicle (PBS) treated EAE mice showed a significant $(* * * p<0.05)$ increase in optic nerve $(N=8)$ inflammation as compared with control $(N=6)$ mouse optic nerves. All AMP cell treatment paradigms $(1 \times \mathrm{IP}, N=6 ; 1 \times \mathrm{IV}, N=$ $7 ; 3 \times \mathrm{IP}, \mathrm{N}=6 ; 3 \times \mathrm{IV}, N=9)$ significantly decreased inflammation in the optic nerves as compared with vehicle-treated EAE mice $\left({ }^{@} p<0.05\right.$; $\left.{ }^{@} p<0.01\right)$. b Images show IBA $1+$ cells in one representative optic nerve section (original magnification $\times 10$, Scale bars: $200 \mu \mathrm{M}$ ). Few microglia/macrophages are observed in the control mouse optic nerve, whereas the vehicle-treated mouse optic nerve contains numerous cells and optic nerves from AMP cell-treated EAE mice contain only moderate

IBA1+ cell levels. c Demyelination in the optic nerve was evaluated by LFB staining and scored using a 3 point scale. Vehicle-treated EAE mice showed a significant $(* * p<0.01)$ increase in optic nerve demyelination as compared with control mouse optic nerves. AMP cell treatment with $1 \times$ IP and IV injection, and $3 \times$ IV injection, significantly decreased demyelination in the optic nerves as compared with vehicle-treated EAE mice $\left({ }^{@} p<0.05 ;{ }^{@}{ }^{@} p<0.01\right)$, and 3× IP injection of AMP cells lead to a non-significant trend in attenuation of myelin loss. $\mathbf{d}$ Representative images of optic nerves stained with LFB show a vehicle-treated EAE mouse with myelin loss in the optic nerve, AMP cell-treated mouse optic nerves with partial preservation of myelin. Original magnification $\times 20$, Scale bars: $200 \mu \mathrm{M}$. Data represent mean \pm SEM of measurements from the right eye of each mouse 
NCT03687632). Therefore, they offer a potential translational pathway to explore their use in treating human MS patients. In the current study design, clinical pathology was assessed at a late time point in the EAE disease, when prior studies have shown that optic nerve neurodegeneration has peaked $[21,25$, 27], in order to assess the effects of AMP cell therapy on demyelination and neurodegeneration; thus, the study was not tailored to determine whether AMP cells exert effects by modulating peripheral immune responses early in the EAE disease, and/or by migrating into the CNS and secreting factors locally to simulate effects of ST266. Immunohistochemical staining of spinal cord sections with anti-human antibodies did reveal several examples of AMP cells integrated into the CNS of treated mice (data not shown), but cells were rare and not found consistently, suggesting that if this mechanism is important, AMP cells may have migrated earlier in the disease

a AMP Cells Decrease OKR Visual Dysfunction

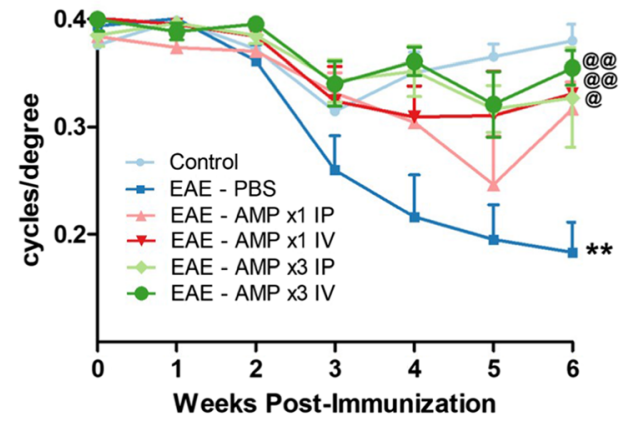

C

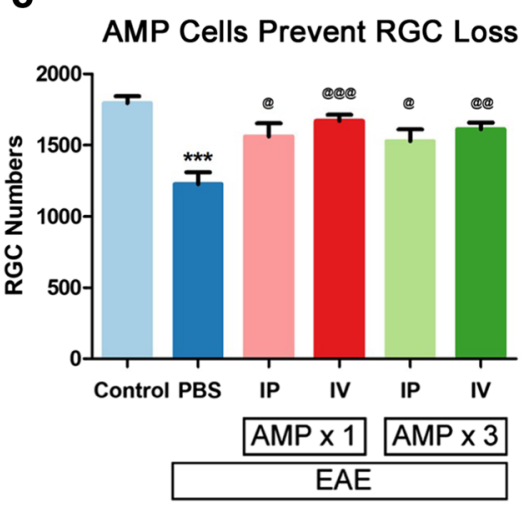

d

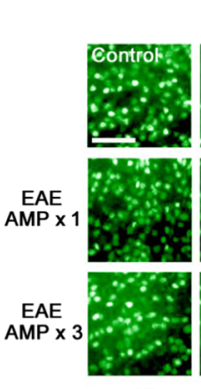

IP

Fig. 4 AMP cells attenuate visual dysfunction and RGC loss. a Visual function was evaluated by OKR weekly. Eyes of vehicle (PBS)-treated EAE mice $(N=8)$ showed a significant $(* * p<0.01)$ progressive decrease in OKR scores over 6 weeks as compared with control $(N=6)$ mouse eyes. $3 \times$ dosing of AMP cells either IP $\left(N=6,{ }^{@} p<0.01\right)$ or IV $(N=9$, $\left.@ \varrho^{@} p 0.01\right)$, as well as $1 \times$ IV AMP cell treatment $\left(N=7,{ }^{@} p<0.05\right)$, significantly attenuated vision loss over 6 weeks, while $1 \times \operatorname{IP}(N=6)$ AMP cell administration induced a non-significant trend in improved OKR responses. b Final visual function measured on day 42 p.i. showed that the significant $(* * *<0.001)$ decrease in OKR responses in vehicletreated EAE mice as compared with control mice was significantly prevented in all AMP cell treatment groups $\left({ }^{@} \mathrm{p}<0.05\right.$; $\left.{ }^{@} \mathrm{p} p<0.01\right)$; $@ p<0.001)$. c To evaluate RGC loss, retinas were isolated at day 42 p.i., stained with Brn3a antibodies, and counted by a masked investigator. course and may have been cleared by the time the mice were euthanized. Nonetheless, it is helpful to consider evidence from prior literature suggesting that both mechanisms, peripheral immune modulation and direct CNS effects, are likely involved. Overexpression of inflammatory cytokines are critically involved in the pathogenesis of EAE and MS and contribute to active inflammatory processes. Studies show that disease progression can be reduced by inhibiting cytokines controlling CD4+ T cell differentiation and expression during the course of EAE [47]. Proinflammatory cytokines also increase blood vessel permeability resulting in an influx of inflammatory cells including auto-reactive $\mathrm{T}$ cells and monocytes resulting in more inflammation, demyelination and tissue damage [29, 48, 49]. Downregulation of pro-inflammatory cytokines and upregulation of anti-inflammatory cytokines results in amelioration of MS/EAE disease progression [50, 51]. Prior studies also

\section{b AMP Cells Prevent Vision Loss at Day 42}

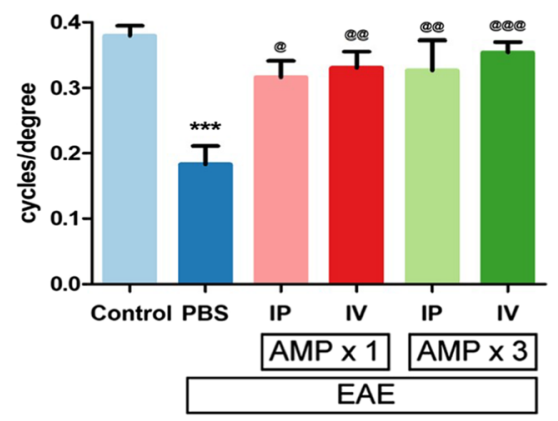

e
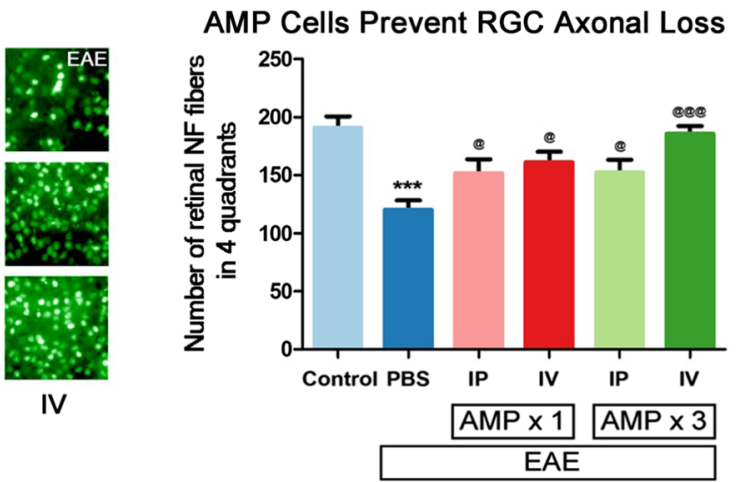

The significant $(* * * p<0.001)$ RGC loss found in vehicle-treated EAE mice as compared with control mice was significantly prevented in all AMP cell treatment groups $\left({ }^{@} p<0.05 ;{ }^{@ @ ~} p<0.01\right)$; $\left.{ }^{@ @} p<0.001\right)$. d Representative images showing RGCs in one field from one retina from each treatment group (original magnification $\times 20$, Scale bars: $50 \mu \mathrm{M}$ ) demonstrate the reduced RGC numbers induced by EAE and prevented by AMP cell treatment. e To evaluate axonal loss in the retina, retinas were isolated at day 42 p.i., stained with Neurofilament RT-97 antibodies and axonal fibers were counted by a masked investigator. Significant $(* * * p<0.001)$ axonal loss found in vehicle-treated EAE mice as compared with control mice was significantly prevented in all AMP cell treatment groups $\left({ }^{@} p<0.05\right.$; $\left.{ }^{@ @ @ ~} p<0.001\right)$. Data represent mean \pm SEM of measurements from the right eye of each mouse 
showed that human amnion mesenchymal stem cell treatment decreased pro-inflammatory cytokines in both the CNS and periphery of EAE mice [52]. Amniotic epithelial cells inhibit both innate and adaptive immune system responses by inhibiting migration of neutrophils and macrophages, inhibiting protease activity and secretion of factors which reduce T-cell and B-cell proliferative responses to mitogens [53]. Similar characteristics of AMP cells may also have contributed in part to the neuroimmunomodulatory effect in our current study. Indeed, AMP cells have been shown to inhibit CMV positive $\mathrm{T}$ cells by an average of $95 \%$, consistent with an immunomodulatory mechanism [19].

Human AECs express neural progenitor cell markers and have putative potentiality of neural stem cells [54]. Amniotic cells express and secrete neurotropic factors which enhance regeneration. Human amniotic membrane itself is known to secrete multiple cytokines and growth factors, including BDNF (brain-derived neurotropic factor) and GDNF (Glial Cell Derived Neurotropic Factor), which play a major role in neuro-regeneration [55]. ST266, the secretome of AMP cells, has been shown to contain physiological levels of PDGF (Platelet Derived Growth Factor), VEGF (Vascular Endothelial Growth Factor), Angiogenin, TGF- $\beta 2$ and TIMP-1 (tissue inhibitor of metalloproteinases-1), which have been implicated in promoting neuronal survival individually or in combination [21, 55-60]. Furthermore, the neuroprotective effects of ST266 involves reduction of oxidative stress, SIRT1-mediated enhancement of mitochondrial function, and pAKT signaling [21]. Consistent with these prior findings of ST266, our current results suggest that AMP cell administration by either IV or IP routes may result in the migration of the cells to the impaired area and induce similar neuroprotective and immunomodulatory functions by locally secreting neurotropic or immunomodulatory factors. Indeed, migration, differentiation and increased expression of growth factors and resultant functional recovery promotion by other amniotic stem cells were exhibited in prior studies [46]. Interestingly, transplantation of amnion cells directly into the CNS induced neuroprotection by increased neurotrophin release even with no morphological integration shown in a model of Parkinson disease [61], suggesting a local effect of secreted factors is a likely mechanism of stem cell-mediated neuroprotection even when such cells are hard to identify.

Together, current results show that both IP and IV administration of single or multiple doses of AMP cells can exert at least some protective effects, although neuroprotective effects of IP treatment were slightly less robust than that of IV treatment, and we surmise this may be due to more efficient distribution of cells following IV administration. This result is similar to studies using mesenchymal stem cells which showed inferior therapeutic effects of IP administration that improved with repeated IP injections [62]. Interestingly, repeated IP injection of AMP cells induced variable levels of improvement in $\mathrm{RGC} / v i s u a l$ preservation and spinal cord EAE disease, suggesting that a higher dose at the single day 9 time point might be more potent than lower IP doses, and indicate that precise dosing regimens require further investigation. Despite being a routine method of administration in clinical practice, IP is less used as an administration route for stem cells [63]; however, studies show that IV injection of stem cells may result in cell aggregation and can produce pulmonary emboli or infarctions [64]; thus, both delivery methods were examined here.

Overall, potent disease suppression and improvement in neurologic function in both optic nerve and spinal cord suggest that AMP cells are a potential novel therapy for MS that warrant continued study. The tremendous neuroprotective properties demonstrated even with a single administration supports AMP cells as a potential easily administered therapy for MS and other autoimmune diseases. Importantly, scientific studies show human AEC transplantation does not induce tumorigenicity [65-67], suggesting that systemic administration of AMP cells is likely a safe cell therapy strategy for MS that delivers a unique and reproducible combination of factors to induce both anti-inflammatory and neuroprotective benefits.

Acknowledgments Funding provided by NIH Grants EY019014 and EY030163; Research to Prevent Blindness; the F. M. Kirby Foundation; the RWJ Harold Amos Faculty Development Award; and Noveome Biotherapeutics, Inc. The RT97 antibody developed by John Wood was obtained from the Developmental Studies Hybridoma Bank developed under the auspices of the NICHD and maintained by the University of Iowa, Department of Biological Sciences (Iowa City, IA).

Required Author Forms Disclosure forms provided by the authors are available with the online version of this article.

\section{References}

1. Goldenberg MM. Multiple sclerosis review. Phar \& Therap 2012;37:175-184

2. Simacek KF, Ko JJ, Moreton D, Varga S, Johnson K, Katic BJ. The Impact of Disease Modifying Therapy Access Barriers on People With Multiple Sclerosis: Mixed-Methods Study. J Med Internet Res 2018; 20:e11168.

3. Frohman EM, Racke MK, Raine CS. Multiple sclerosis-the plaque and its pathogenesis. N Engl J Med 2006; 354:942.

4. Kale N. Optic neuritis as an early sign of multiple sclerosis. Eye Brain 2016; 8:195-202.

5. You Y, Barnett MH, Yiannikas C, Parratt J, Matthews J, Graham SL, Klistorner A. Chronic demyelination exacerbates neuroaxonal loss in patients with MS with unilateral optic neuritis. Neurol Neuroimmunol Neuroinflamm 2020; 7: e700.

6. Khan RS, Baumann B, Dine K, Song Y, Dunaief JL, Kim SF, Shindler KS. Dexras1 Deletion and Iron Chelation Promote Neuroprotection in Experimental Optic Neuritis. Sci Rep 2019; 9: 11664.

7. Ontaneda D, Rae-Grant AD. Management of acute exacerbations in multiple sclerosis. Ann Indian Acad Neurol 2009; 12: 264-272. 
8. Richert ND, Ostuni JL, Bash CN, Leist TP, McFarland HF, Frank $\mathrm{JA}$. Interferon beta-1b and intravenous methylprednisolone promote lesion recovery in multiple sclerosis. Mult Scler 2001; 7:4958.

9. Beck RW, Cleary PA, Trobe JD, Kaufman DI, Kupersmith MJ, Paty DW, Brown CH. The effect of corticosteroids for acute optic neuritis on the subsequent development of multiple sclerosis. The Optic Neuritis Study Group. N Engl J Med 1993; 329:1764-1769.

10. Brusaferri F, Candelise L. Steroids for multiple sclerosis and optic neuritis: a meta-analysis of randomized controlled clinical trials. J Neurol 2000; 247:453-442.

11. Goodin DS. Interferon beta treatment for multiple sclerosis. Lancet. 1999; 353:9151.

12. Beck RW, Gal RL. Treatment of acute optic neuritis: a summary of findings from the optic neuritis treatment trial. Arch Ophthalmol 2008; 126:994-995.

13. Optic Neuritis Study Group. Multiple sclerosis risk after optic neuritis: final optic neuritis treatment trial follow-up. Arch Neurol 2008; 65:727-732

14. Diem R, Hobom M, Maier K, Weissert R, Storch MK, Meyer R, Bähr M. Methylprednisolone increases neuronal apoptosis during autoimmune CNS inflammation by inhibition of an endogenous neuroprotective pathway. J Neurosci 2003; 23:6993-7000.

15. Cuascut FX, Hutton GJ. Stem Cell-Based Therapies for Multiple Sclerosis: Current Perspectives. Biomedicines. 2019; 7: 26.

16. Scolding NJ, Pasquini M, Reingold SC, Cohen JA. Cell-based therapeutic strategies for multiple sclerosis: International Conference on Cell-Based Therapies for Multiple Sclerosis. Brain. 2017; 140: 2776-2796.

17. Jafarzadeh-Bejargafshe M, Hedayati M, Zahabiasli S, Tahmasbpour E, Rahmanzadeh S, Nejad-Moghaddam A. Saftey and efficacy of stem cell therapy for treatment of neural damage in patients with multiple sclerosis. Stem Cell Investig 2019; 6:44.

18. McDonald CA, Payne NL, Sun G, Moussa L, Siatskas C, Lim R, Wallace EM, Jenkin G, Bernard CC. Immunosuppressive potential of human amnion epithelial cells in the treatment of experimental autoimmune encephalomyelitis. J Neuroinflammation 2015; 12: 112.

19. Banas RA, Trumpower C, Bentlejewski C, Marshall V, Sing G, Zeevi A. Immunogenicity and immunomodulatory effects of amnion-derived multipotent progenitor cells. Hum Immunol 2008; 69:321-328

20. Banas R, Miller C, Guzik L, Zeevi A. Amnion derived multipotent progenitor cells inhibit blood monocyte differentiation into mature dendritic cells. Cell Transplant 2014; 23: 1111-1125.

21. Khan RS, Dine K, Bauman B, Lorentsen M, Lin L, Brown H, Hanson LR, Svitak AL, Wessel H, Brown L, Shindler KS. Intranasal Delivery of A Novel Amnion Cell Secretome Prevents Neuronal Damage and Preserves Function In A Mouse Multiple Sclerosis Model. Sci Rep 2017; 7:41768.

22. Khan RS, Dine K, Geisler JG, Shindler KS. Mitochondrial Uncoupler Prodrug of 2,4-Dinitrophenol, MP201, Prevents Neuronal Damage and Preserves Vision in Experimental Optic Neuritis. Oxid Med Cell Longev. 2017; 2017:7180632.

23. Khan RS, Dine K, Luna E, Ahlem C, Shindler KS. HE3286 reduces axonal loss and preserves retinal ganglion cell function in experimental optic neuritis. Invest Ophthalmol Vis Sci 2014; 55:57445751.

24. Prusky GT, Alam NM, Beekman S, Douglas RM. Rapid quantification of adult and developing mouse spatial vision using a virtual optomotor system. Invest Ophthalmol Vis Sci 2004; 45: 46114616.

25. Quinn T, Dutt M, Shindler KS. Optic neuritis and retinal ganglion cell loss in a chronic murine model of multiple sclerosis. Front Neurol 2011; 2: 50.
26. Shindler KS, Ventura E, Dutt M, Elliott P, Fitzgerald DC, Rostami A. Oral resveratrol reduces neuronal damage in a model of multiple sclerosis. J Neuroophthalmol 2010; 30:328-339.

27. Fonseca-Kelly Z, Nassrallah M, Uribe J, Khan RS, Dine K, Dutt M, Shindler KS. Resveratrol neuroprotection in a chronic mouse model of multiple sclerosis. Front Neurol 2012; 3:84.

28. Singh M, Khan RS, Dine K, Das Sarma J, Shindler KS. Intracranial Inoculation Is More Potent Than Intranasal Inoculation for Inducing Optic Neuritis in the Mouse Hepatitis Virus-Induced Model of Multiple Sclerosis. Front Cell Infect Microbiol 2018; 8:311.

29. Constantinescu CS, Farooqi N, O’Brien K, Gran B. Experimental autoimmune encephalomyelitis (EAE) as a model for multiple sclerosis. Br J Pharmacol 2011; 164:1079-1106.

30. Day M. Histopathology of EAE. Experimental Models of Multiple Sclerosis. L E and C C. New York, Springer, 2005; pp. 25-43.

31. Pierson E, Simmons SB, Castelli L, Goverman JM. Mechanisms regulating regional localization of inflammation during CNS autoimmunity. Immunol Rev 2012; 248:205-215.

32. Greter M, Heppner FL, Lemos MP, Odermatt BM, Goebels N, Laufer T, Noelle RJ, Becher B. Dendritic cells permit immune invasion of the CNS in an animal model of multiple sclerosis. Nat Med 2005; 11:328-334.

33. Choi BY, Kim JH, Kho AR, Kim IY, Lee SH, Lee BE, Choi E, Sohn M, Stevenson M, Chung TN, Kauppinen TM, Suh SW. Inhibition of NADPH oxidase reduces EAE-induced white matter damage in mice. J Neuroinflammation 2015; 12:104.

34. Wu GF, Parker Harp CR, Shindler KS. Optic Neuritis: A Model for the Immuno-pathogenesis of Central Nervous System Inflammatory Demyelinating Diseases. Curr Immunol Rev 2015; 11: 85-92.

35. Manogaran P, Samardzija M, Schad AN, Wicki CA, Walker-Egger C, Rudin M, Grimm C, Schippling S. Retinal pathology in experimental optic neuritis is characterized by retrograde degeneration and gliosis. Acta Neuropathol Commun 2019; 7:116.

36. Guy J. Optic nerve degeneration in experimental autoimmune encephalomyelitis. Ophthalmic Res 2008; 40:212-216.

37. Tian AY, Zhang RW, Shi XG, Yu HM. Alteration of T helper cell subsets in the optic nerve of experimental autoimmune encephalomyelitis. Int J Mol Med 2010; 25:869-874.

38. Horstmann L, Kuehn S, Pedreiturria X, Haak K, Pfarrer C, Dick HB, Kleiter I, Joachim SC. Microglia response in retina and optic nerve in chronic experimental autoimmune encephalomyelitis. J Neuroimmunol 2016; 298:32-41.

39. Kubo M, Sonoda Y, Muramatsu R, Usui M. Immunogenicity of human amniotic membrane in experimental xenotransplatation. Invest Ophthalmol Vis Sci 2001; 42:1539.

40. Lefebvre S, Adrian F, Moreau P, Gourand L, Dausset J, BerrihAknin S, Carosella ED, Paul P. Modulation of HLA-G expression in human thymic and amniotic epithelial cells. Hum Immunol. 2000; 61:1095.

41. Houlihan J, Biro PA, Harper H, Jenkinson H, Holmes C. The human amnion is a site of MHC class Ib expression: Evidence for the expression of HLA-E and HLA-G. J Immunol 1995; 154:5665.

42. Le Rond S, Le Maoult, Creput C, Menier C, Deschamps M, Le Friec G, Amiot L, Durrbach A, Dausset J, Carosella ED, RouasFreiss N. Alloreactive CD4+ and CD8+ T cells express the immunotolerant HLA-G molecule in mixed lymphocyte reactions: in vivo implications in transplanted patients. Eur J Immunol. 2004; 34:649-660

43. Le Rond S, Azéma C, Krawice-Radanne I, Durrbach A, Guettier C, Carosella ED, Rouas-Freiss N. Evidence to support the role of HLA-G5 in allograft acceptance through induction of immunosuppressive/ regulatory T cells. J Immunol 2006; 176: 3266-3276.

44. Naji A, Durrbach A, Carosella E, Rouas-Freiss N. Soluble HLA-G and HLA-G1 expressing antigen-presenting cells inhibit T-cell 
alloproliferation through ILT-2/ILT-4/FasL-mediated pathways. Hum Immunol 2007; 68:233.

45. Liu YH, Vaghjiani V, Tee JY, To K, Cui P, Oh DY, Manuelpillai $\mathrm{U}$, Toh BH, Chan J. Amniotic epithelial cells from the human placenta potently suppress a mouse model of multiple sclerosis. PLoS One 2012; 7:e35758.

46. Abbasi-Kangevari M, Ghamari SH, Safaeinejad F, Bahrami S, Niknejad H. Potential Therapeutic Features of Human Amniotic Mesenchymal Stem Cells in Multiple Sclerosis: Immunomodulation, Inflammation Suppression, Angiogenesis Promotion, Oxidative Stress Inhibition, Neurogenesis Induction, MMPs Regulation, and Remyelination Stimulation. Front Immunol 2019; 10:238.

47. Chu CQ, Wittmer S, Dalton DK. Failure to suppress the expansion of the activated cd4 $\mathrm{T}$ cell population in interferon gamma-deficient mice leads to exacerbation of experimental autoimmune encephalomyelitis. J Exp Med 2000; 192:123-128.

48. McCarthy DP, Richards MH, Miller SD. Mouse models of multiple sclerosis: Experimental autoimmune encephalomyelitis and Theiler's virus-induced demyelinating disease. Methods Mol Biol 2012; 900: 381-401

49. Benveniste EN. Role of macrophages/microglia in multiple sclerosis and experimental allergic encephalomyelitis. J Mol Med 1997; 75:165-173.

50. Martins TB, Rose JW, Jaskowski TD, Wilson AR, Husebye D, Seraj HS, Hill HR. Analysis of proinflammatory and antiinflammatory cytokine serum concentrations in patients with multiple sclerosis by using a multiplexed immunoassay. Am J Clin Pathol 2011; 136:696-704.

51. Jahan-Abad AJ, Karima S, Shateri S, Baram SM, Rajaei S, Morteza-Zadeh P, Borhani-Haghighi M, Salari AA, Nikzamir A, Gorji A. Serum pro-inflammatory and anti-inflammatory cytokines and the pathogenesis of experimental autoimmune encephalomyelitis. Neuropathology. 2020; 40:84-92.

52. Shu J, He X, Li H, Liu X, Qiu X, Zhou T, Wang P, Huang X. The Beneficial Effect of Human Amnion Mesenchymal Cells in Inhibition of Inflammation and Induction of Neuronal Repair in EAE Mice. J Immunol Res 2018; 24:5083797.

53. Haochuan Li, Jerry Y, Niederkorn, Neelam S, Mayhew E, Ann Word R, James P, McCulley, Alizadeh H. Immunosuppressive Factors Secreted by Human Amniotic Epithelial Cells. Invest Ophthalmol Vis Sci. 2005; 46:900-907.

54. Sakuragawa N, Thangavel R, Mizuguchi M, Hirasawa M, Kamo I. Expression of markers for both neuronal and glial cells in human amniotic epithelial cells. Neurosci Lett 1996; 209:9-12.

55. Banerjee A, Nürnberger S, Hennerbichler S, Riedl S, Schuh CM, Hacobian A, Teuschl A, Eibl J, Redl H, Wolbank S. In toto differentiation of human amniotic membrane towards the Schwann cell lineage. Cell Tissue Bank 2014;15:227-239.

56. Zheng L, Ishii Y, Tokunaga A, Hamashima T, Shen J, Zhao QL, Ishizawa S, Fujimori T, Nabeshima Y, Mori H, Kondo T, Sasahara
M. Neuroprotective effects of PDGF against oxidative stress and the signaling pathway involved. J Neurosci Res 2010; 88:12731284.

57. Sun Y, Jin K, Xie L, Childs J, Mao XO, Logvinova A, Greenberg DA. VEGF- induced neuroprotection, neurogenesis, and angiogenesis after focal cerebral ischemia. J Clin Invest 2003; 111: 18431851.

58. Hoang TT, Johnson DA, Raines RT, Johnson JA. Angiogenin activates the astrocytic Nrf2/antioxidant-response element pathway and thereby protects murine neurons from oxidative stress. J Biol Chem 2019; 294:15095-15103.

59. Polazzi E, Altamira LE, Eleuteri S, Barbaro R, Casadio C, Contestabile A, Monti B. Neuroprotection of microglial conditioned medium on 6-hydroxydopamine-induced neuronal death: role of transforming growth factor beta-2. J Neurochem 2009; 110:545-556

60. Ashutosh, Chao C, Borgmann K, Brew K, Ghorpade A. Tissue inhibitor of metalloproteinases-1 protects human neurons from staurosporine and HIV-1-induced apoptosis: mechanisms and relevance to HIV-1-associated dementia. Cell Death Dis. 2012; 3:e332.

61. Kong XY, Cai Z, Pan L, Zhang L, Shu J, Dong YL, Yang N, Li Q, Huang XJ, Zuo PP. Transplantation of human amniotic cells exerts neuroprotection in MPTP-induced Parkinson disease mice. Brain Res 2008; 1205:108-115.

62. Zickri MB, Fadl SG, Metwally HG. Comparative Study between Intravenous and Intraperitoneal Stem Cell Therapy in Amiodarone Induced Lung Injury in Rat. Int J Stem Cells 2014; 7:1-11.

63. Ghionzoli M, Cananzi M, Zani A, Rossi CA, Leon FF, Pierro A, Eaton S, De Coppi P. Amniotic fluid stem cell migration after intraperitoneal injection in pup rats: implication for therapy. Pediatr Surg Int 2010; 26:79-84.

64. Prockop DJ, Olson SD. Clinical trials with adult stem/progenitor cells for tissue repair: let's not overlook some essential precautions. Blood. 2007; 109:3147-3151.

65. Huiming Xu, Jiaofei Zhang, Kam Sze Tsang, Hao Yang, WeiQiang Gao. Therapeutic Potential of Human Amniotic Epithelial Cells on Injuries and Disorders in the Central Nervous System. Stem Cells Int. 2019; 2019:5432301.

66. Yang PJ, Yuan WX, Liu J, Li JY, Tan B, Qiu C, Zhu XL, Qiu C, Lai DM, Guo LH, Yu LY. Biological characterization of human amniotic epithelial cells in a serum-free system and their safety evaluation. Acta Pharmacol Sin 2018; 39: 1305-1316.

67. Ilancheran S, Michalska A, Peh G, Wallace EM, Pera M, Manuelpillai U. Stem cells derived from human fetal membranes display multilineage differentiation potential. Biol Reprod 2007; 77: $577-588$.

Publisher's Note Springer Nature remains neutral with regard to jurisdictional claims in published maps and institutional affiliations. 